\title{
JOINT PRODUCTION AND SUPPLY CONTROL IN THREE LEVELS FLEXIBLE MANUFACTURING SYSTEMS
}

\author{
HAJJI A. ${ }^{\text {a }}$, GHARBI A. ${ }^{\text {a }}$, KENNE J. P. ${ }^{\text {b }}$ \\ University of Québec, École de Technologie Supérieure, \\ 1100, Notre Dame Ouest, Montréal, Que., Canada H3C 1 K3 \\ a Department of Automated Production Engineering, LCCSP Laboratory \\ $b$ Department of Mechanical Engineering, LITP Laboratory
}

\begin{abstract}
This paper considers a stochastic optimal control problem of a three stages Flexible Manufacturing System. The supplier (i.e., upstream FMS) and the transformation stage (i.e., FMS) are both subject to random events. Our objective is to find a feedback control policy for the supply and production activities that minimizes the incurred cost. It is shown that the considered joint production and supply control problem is difficult to tackle using a dissociated analytical approach. A simulation based approach is thus proposed to achieve a close approximation of the optimal policy. The advantages of the approach include possible extensions after numerical characterization of the optimal control policy.
\end{abstract}

Keywords: Feedback control, optimal control theory, simulation, DOE, FMS, unreliable supply

\section{INTRODUCTION}

An important class of stochastic manufacturing systems faces unreliable upstream supply. In this paper, we consider this class of systems through a three stage flexible manufacturing system responding to a one part type demand.

A review of the relevant control theory literature has showed that the two problems aiming to control production or supply activities lead to different kinds of policies. To control the flow rates of parts through a Flexible Manufacturing System (FMS) Kimemia and Gershwin (1983) introduced the hedging point policy (HPP). Within such a policy, a non negative production surplus of part types, corresponding to the optimal inventory levels, is maintained during times of excess capacity availability to hedge against future capacity shortage caused by machine failures. Based on the pioneering work of Kimemia and Gershwin (1983) and the HPP concept, different classes of manufacturing systems have been investigated. Among many others, Akella and Kumar (1986) developed an explicit formulation of the HPP; Boukas and Haurie (1990) investigated the production and preventive maintenance control problem; Feng and Yan (2000) focused their contribution on providing a suitable production policy for unreliable systems facing stochastic demands; Hajji et al. (2004) developed a production and setup policy for unreliable manufacturing systems; Gharbi and Kenne (2003) provided a suboptimal control policy for the multiple parts multiple machines problem.

The main assumption made in these papers is that the system will never be starved and thus have a reliable supply of raw material. This assumption could simply not be realistic considering the fact that an unreliable supplier or a random delay leads to a random availability of the raw material.

On the other hand, based on different set of assumptions (e.g., deterministic lead time), many works have considered the stochastic aspect of supply. Among others, Bensoussan et al. (1983), Gullu et al. (1999) and Cheng and Sethi (1999). Basically, the dynamic programming approach was employed using the concept of K-convexity to establish the optimality conditions. In the aforementioned works, different proofs of the optimality of (s, S) type policy were provided. Within such a policy an economic lot of raw material is ordered when the upstream inventory level rich s.

In the control literature, these two problems leading to the HPP and (s, S) policies are still considered independently. This is due, in part, to the difficulty behind the mathematical formulation of the whole system dynamic. However, the latest literature has shown that the integrated models through the intra- 
department planning by integrating raw material procurement and its production is more realistic and will result to better performance than that when the planning is performed separately (Lee, 2005).

The main contribution of this paper is to develop an integrated production and supply policy of a stochastic three stages Flexible Manufacturing System. A stochastic dynamic programming problem is formulated. The structure of the solution, under appropriate conditions, is obtained by using the fact that the value function is the unique viscosity solution to the associated Hamilton Jacobi Bellman equations (HJB). Owing that an analytical solution of HJB equations is not in general available; a numerical approach is adopted to illustrate the structure of the control policy. A simulation based experimental approach is then adopted to achieve a close approximation of the optimal control policy.

The paper is organized as follows. Section 2 presents the statement of the optimal production and supply problem. Section 3 presents the numerical results and the related control policy. Section 4 describes the simulation based experimental approach used to quantify, achieve an approximation of the optimal policy and to determine the related cost incurred for the integrated policies. Section 5 presents a decisional process offering useful solution of the quantified feedback policy. The paper is concluded in section 6 .

\section{PROBLEM STATEMENT}

The flexible manufacturing system under study (figure 1) consists of an unreliable system supplied by an unreliable upstream supplier. The whole system faces a one family product demand.

The state of the system at time $t$ has three components including:

- A continuous part which describes the cumulative surplus vector (inventory if positive and backlog if negative) and measured by $x_{2}(\mathrm{t})$.

- A combined part which describes the raw material level and measured by $x_{1}(\mathrm{t})$. This part faces the continuous downstream demand and an impulsive upstream supply when a $Q_{i}$ lot of raw material is received at instant $\theta_{i}$. Even if it is available, the FMS can not proceed parts when $x_{1}(\mathrm{t})$ is equal to zero. Let $0 \leq x_{1}(\mathrm{t}) \leq L$ be the capacity constraint of the raw material stock.

- A discrete part which describes the system (supplier and FMS) state and denoted by $\xi(t)$. This is a stochastic process taking values in $\mathrm{M}=\{1,2,3,4\} . \zeta(t)=1 \Rightarrow$ Both the supplier and the FMS are available. $\zeta(t)=2(3) \Rightarrow$ The supplier (respect. the FMS) is unavailable. $\zeta(t)=4 \Rightarrow$ Both the supplier and the FMS are unavailable.

For the considered manufacturing system, the state space is given by $\left(x_{1}, x_{2}, \alpha\right)$ such that:

$$
x_{1} \in[0, L] ; x_{2} \in R ; \alpha \in M=\{1,2,3,4\}
$$

The dynamic of the stock levels $x_{1}(\mathrm{t})$ and $x_{2}(\mathrm{t})$ is given by the following differential equations.

$$
\begin{aligned}
& \dot{x}_{2}(t)=u(t, \alpha)-d, x_{2}(0)=x_{2}, \forall t \geq 0 \\
& \left.\dot{x}_{1}(t)=-u(t, \alpha), x_{1}(0)=x_{1}, \forall t \in\right] \theta_{i}, \theta_{i+1}[ \\
& x_{1}\left(\theta_{i}^{+}\right)=x_{1}\left(\theta_{i}^{-}\right)+Q_{i}(\alpha), i=1, \ldots, N .
\end{aligned}
$$

Where $x_{1}, x_{2}$ denote the stocks levels at time $\mathrm{t}=0$. $\mathrm{x}_{1}(t), \mathrm{x}_{2}(t)$, denote the raw material and finish product stock levels at time $t, d$ the demand rate and $u(\mathrm{t}, \alpha)$ the FMS production rate. $\theta_{i}^{-}, \theta_{i}^{+}$, denote negative and positive boundaries of the $N$ receipt instants $i$.

FMS and supplier available and unavailable times are assumed to evolve according to a continuous time Markov processes with modes in $M_{1}=\{1,2\}$ and $M_{2}=\{1,2\}$ and with transition rates matrix $\mathrm{T}_{1}$ and $\mathrm{T}_{2}$ such that $\mathrm{T}_{i}=\left\{q_{\alpha \beta}^{i}\right\}$, with $q_{\alpha \beta}^{i} \geq 0 \quad$ if $\quad \alpha \neq \beta \quad$ and $q_{\alpha \alpha}^{i}=-\sum_{\beta \neq \alpha} q_{\alpha \beta}^{i}$, where $\alpha, \beta \in M_{i}$. The transitions rates matrix $\mathrm{T}_{i}$ is then expressed as follows:

$$
\mathrm{T}_{i}=\left|\begin{array}{cc}
-q^{i}{ }_{12} & q^{i}{ }_{12} \\
q^{i}{ }_{21} & -q^{i}{ }_{21}
\end{array}\right|
$$

At any given time, the production rates and the order quantities have to satisfy the production and supply capacity constraints.

$$
\begin{aligned}
& 0 \leq u(t, \alpha) \leq U_{\max } \times \operatorname{Ind}\{\zeta(t)=1,2\} \\
& 0 \leq Q_{i}(t) \leq L \times \operatorname{Ind}\{\zeta(t)=1,3\}, i=1, \ldots, N
\end{aligned}
$$

Where, $\operatorname{Ind}\{\zeta(t)=\alpha\}=\left\{\begin{array}{cc}1, & \text { if } \zeta(t)=\alpha \\ 0, & \text { otherwise }\end{array}\right.$, $U_{\max }$ denotes the maximal production rate, and $\mathrm{L}$ the raw material buffer capacity. 


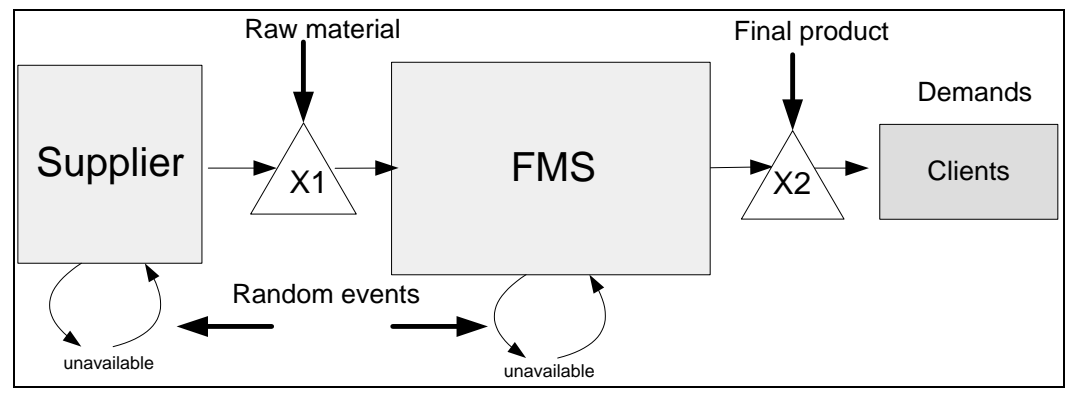

Fig. 1. Unreliable three stages FMS.

Our decision variables are the production rate $\mathrm{u}(\cdot)$ and a sequence of supply orders denoted by $\Omega=\left\{\left(\theta_{0}, Q_{0}\right),\left(\theta_{1}, Q_{1}\right), \ldots\right\}$, with $\left(\theta_{i}, Q_{i}\right)$ defined by the time $\theta_{i}$ at witch the order is placed and the order quantity $Q_{i}$. Given (2), let $A(\alpha)$ the set of admissible decisions $(\Omega, \boldsymbol{u}()$.$) given by:$

$A(\alpha)=\left\{\begin{array}{l}(\Omega, u(.)): 0 \leq u(t, \alpha) \leq U_{\text {max }} \times \text { Ind }\{\zeta(t)=1,2\} ; \\ 0 \leq Q_{i}(t) \leq L \times \operatorname{Ind}\{\zeta(t)=1,3\}\end{array}\right\}$

The instantaneous production, finished product inventory and backlog cost function $g($.) is given by the following equation:

$$
\begin{aligned}
& g\left(x_{1}(t), x_{2}(t), u(t, \alpha)\right)= \\
& \left.c_{1}^{+} \cdot x_{1}^{+}+c_{2}^{+} \cdot x_{2}^{+}+c_{2}^{-} \cdot x_{2}^{-}+c_{u} \cdot u(\cdot), t \in\right] \theta_{i}, \theta_{i+1}[
\end{aligned}
$$

Where, $x_{i}^{+}=\max \left(0, x_{i}\right)$ and $x_{i}^{-}=\max \left(-x_{i}, 0\right)$.

$c_{2}^{+}$and $c_{2}^{-}$denote the inventory and backlog costs of the finish product, $c_{1}^{+}$and $c_{u}$ are the inventory cost of the raw material and the production cost.

The instantaneous cost function of the supply order at time $\theta_{i}$, is given by the following equation:

$$
\begin{aligned}
& R\left(Q_{i}, \alpha\right)=K \operatorname{Ind}\left\{t=\theta_{i}\right\}+c_{A} Q_{i}+c_{1}^{+} x_{1}^{+}\left(\theta_{i}\right) \\
& +c_{2}^{+} x_{2}^{+}\left(\theta_{i}\right)+c_{2}^{-} x_{2}^{-}\left(\theta_{i}\right)+c_{u} u\left(\theta_{i}\right)
\end{aligned}
$$

Where $K$ and $c_{A}$ are the order and raw material cost. Using (3)-(4), the total cost $J($.) can be defined by the following expression:

$$
\begin{aligned}
& J\left(x_{1}, x_{2}, u, \theta, Q, \alpha\right)= \\
& E\left[\int_{0}^{\infty} e^{-\rho t} g\left(x_{1}, x_{2}, u\right) d t+\sum_{l=0}^{\infty} e^{-\rho \theta_{i}}\left(K+c_{A} \times Q_{i}\right)\right]
\end{aligned}
$$

Where $\rho$ denotes the discounted rate.

It is interesting to note that the discounted rate is used to update an amount $\mathrm{M}$ between two points in the horizon. In fact, it is common when the amounts are affected on a continuous basis to sum the actual values as follows:

$$
\int_{t 1}^{t 2} M e^{-\rho t} d t
$$

The production planning problem considered herein is to find an admissible decision or control policy $(\Omega$, $\boldsymbol{u}($.$) ) that minimizes J($.$) given by (5) considering$ equations (1) to (3). This is a feedback control that specifies the control actions when the system is in a given state $\left(x_{1}, x_{2}, \alpha\right)$. The feedback control determines the production rate and the supply decisions as a function of the system state.

The corresponding value function $v($.) can be given by the following:

$$
v\left(x_{1}, x_{2}, \alpha\right)=\min _{(\Omega, u) \in A} J\left(x_{1}, x_{2}, u, \Omega, \alpha\right)
$$

As in Sethi and Zhang (1994), and using the optimal impulsive control theory (Sethi and Thompson, 2000) it can be shown that the value function $v\left(x_{1}, x_{1}, \alpha\right)$ is the unique viscosity solution to the following $\mathrm{HJB}$ equation.

$\min \left\{\begin{array}{c}\min _{u}\left\{+\sum_{\beta \neq \alpha} \lambda_{\alpha \beta}\left(v\left(x_{1}, x_{2}, \beta\right)-v\left(x_{1}, x_{2}, \alpha\right)\right)\right\} \\ -\rho v\left(x_{1}, x_{2}, \alpha\right) \\ \min _{Q}\left\{R(Q, \alpha)+v\left(x_{1}+Q, x_{2}+\varepsilon^{\prime}, \alpha\right)\right\}-v\left(x_{1}, x_{2}, \alpha\right)\end{array}\right\}=0$

Where $(v)_{x}($.$) , denotes the gradients of v($.$) with$ respect to $\boldsymbol{x}$.

The production and supply policy that we are seeking is obtained when the value function is known. While we cannot solve analytically the HJB equations (7), we can apply numerical methods to obtain the approximation of the value function and the associated control policy.

\section{NUMERICAL RESULTS}

In this section we present the numerical results and the obtained optimal control policy for the considered system.

The numerical methods used to solve the optimality conditions, corresponding to the stochastic optimal 
control problem, are based on the Kushner approach (Kushner and Dupuis, 1992). The solution of the numerical approximation of $v_{i}(x, \alpha)$ may be obtained by either successive approximation or policy improvement techniques (Boukas and Haurie, 1990 and Kushner and Dupuis, 1992).

The implementation of the approximation technique needs the use of a finite grid denoted herein $G_{h}$, where $h$ is a given vector of finite difference intervals. The successive approximation algorithm is given by the following 6 steps for a given finite difference intervals $h$.

Step 1. (Initialization) Choose $\delta \in R^{+}$; set n: $=1$, and $\left(v^{h}\left(x_{1}, x_{2}, \alpha\right)\right)^{n}:=0, \forall \alpha \in M, \forall x_{1}, x_{2} \in G_{h}$

Step 2. Set

$\left(v^{h}\left(x_{1}, x_{2}, \alpha\right)\right)^{n-1}:=\left(v^{h}\left(x_{1}, x_{2}, \alpha\right)\right)^{n}, \forall \alpha \in M, \forall x_{1}, x_{2} \in G_{h}$

Step 3. Compute the corresponding cost function

from first part of (7). Obtain $u^{n}($.)

Step 4. Compute the corresponding cost function from second part of (7). Obtain $Q^{n}($.)

Step 5. Compute the corresponding cost function from (7). Obtain $u^{n}($.$) and Q^{n}($.$) for the given$ value function. $\forall \alpha \in M, \forall x_{1}, x_{2} \in G_{h}$ )

Step 6. Test

$$
\begin{aligned}
& \bar{c}:=\min _{x_{1}, x_{2} \in G}\left[\left(v^{h}\right)^{n}\left(x_{1}, x_{2}, \alpha\right)-\left(v^{h}\right)^{n-1}\left(x_{1}, x_{2}, \alpha\right)\right] \\
& \underline{c}:=\max _{x, y \in G}\left[\left(v^{h}\right)^{n}\left(x_{1}, x_{2}, \alpha\right)-\left(v^{h}\right)^{n-1}\left(x_{1}, x_{2}, \alpha\right)\right] \\
& c_{\min }:=\frac{\rho}{1-\rho} \bar{c} \quad c_{\max }:=\frac{\rho}{1-\rho} \underline{c} \\
& \text { if }\left|c_{\max }-c_{\min }\right| \leq \delta \quad \text { then } \\
& \text { stop, }\left(u^{*}, Q^{*}\right)=\left(u^{n}, Q^{n}\right), \\
& \text { else let } \mathrm{n}=\mathrm{n}+1 \quad \text { and go to step } 2
\end{aligned}
$$

Recall that when the supplier is unavailable the FMS has to wait for a random length of time (random delay) for the supplier to become available. Based on this fact and for a best characterization of the policy, several cases of supplier availability have been studied. These cases of study (i.e., supplier availability) showed us the reaction of the FMS facing such situation.

The transition rate matrixes defining the FMS and supplier availabilities are as follow:

$$
\begin{gathered}
T^{1}=\left\lfloor\begin{array}{cc}
-0.2 & 0.2 \\
0.1 & -0.1
\end{array}\right] \quad ; \quad T^{2}=\left\lfloor\begin{array}{cc}
-0.01 & 0.01 \\
0.1 & -0.1
\end{array}\right\rfloor \\
T=\left\lfloor\begin{array}{cccc}
-0.21 & 0.01 & 0.2 & 0 \\
0.1 & -0.3 & 0 & 0.2 \\
0.1 & 0 & -0.11 & 0.01 \\
0 & 0.1 & 0.1 & -0.2
\end{array}\right\rfloor
\end{gathered}
$$

Table 1 shows the constant data for the numerical example.

$\underline{\text { Table } 1 \text { Constant data parameters }}$

\begin{tabular}{ll} 
Parameters & Values \\
\hline$U_{\max }$ & 2.5 \\
$d$ & 2 \\
$\rho$ & 0.4 \\
\hline
\end{tabular}

To ensure a clear characterization of the control policy, several elements were taken into consideration as part of the implementation process. Indeed, the production and supply policies are each observed separately. For each policy, the relevant significant threshold levels are analyzed independently of the others. For each numerical result, the policies are provided as shown in Figure 2a, 2b. $u(t, 1)$ and $u(t, 3)$ are the production policies of the FMS in the system state 1 (FMS and supplier available) and 3 (FMS available and supplier unavailable).

$\Omega\left(x_{1}, x_{2}, 1\right)$ and $\Omega\left(x_{1}, x_{2}, 2\right)$ are the supply policies in system state 1 and 2 (Figure $2 \mathrm{c}$ and $2 \mathrm{~d}$ ).

It follows from our numerical results that the resulting production policy divides the surplus space into two mutually exclusive regions. In region I, we produce at the maximal rate and in region II we have to set the production rate to zero. At the boundary of these regions we have to set the production rate equal to the demand rate. Moreover, the results show that the supply policy is governed by an order quantity (illustrated by region III in figure 2c) and an order point (illustrated by region IV in figure $2 \mathrm{c}$ ). This order point reflects the necessity to have a security raw material stock level to face a possible random delivery delay when the supplier is unavailable. In conclusion, the optimal policy is a combination of the Hedging Point Policy and an (s, Q) type inventory Policy. Let s, Q and $\mathrm{Z}$ define the policy parameters.

After several sensitivity analysis, we have clearly observed that the results obtained make sense, and that the structure of the policy defined by the 3 parameters (s, Q and Z) is always maintained. This allows the development of a parameterized production and supply control policy defined by the following equations:

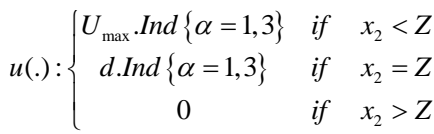

$$
\begin{aligned}
& \Omega(.): \begin{cases}Q & \text { if } X_{1} \leq s \\
0 & \text { otherwise }\end{cases}
\end{aligned}
$$

With the following constraints:

$$
Z \geq 0 ; s<Q<L ; s \geq 0
$$




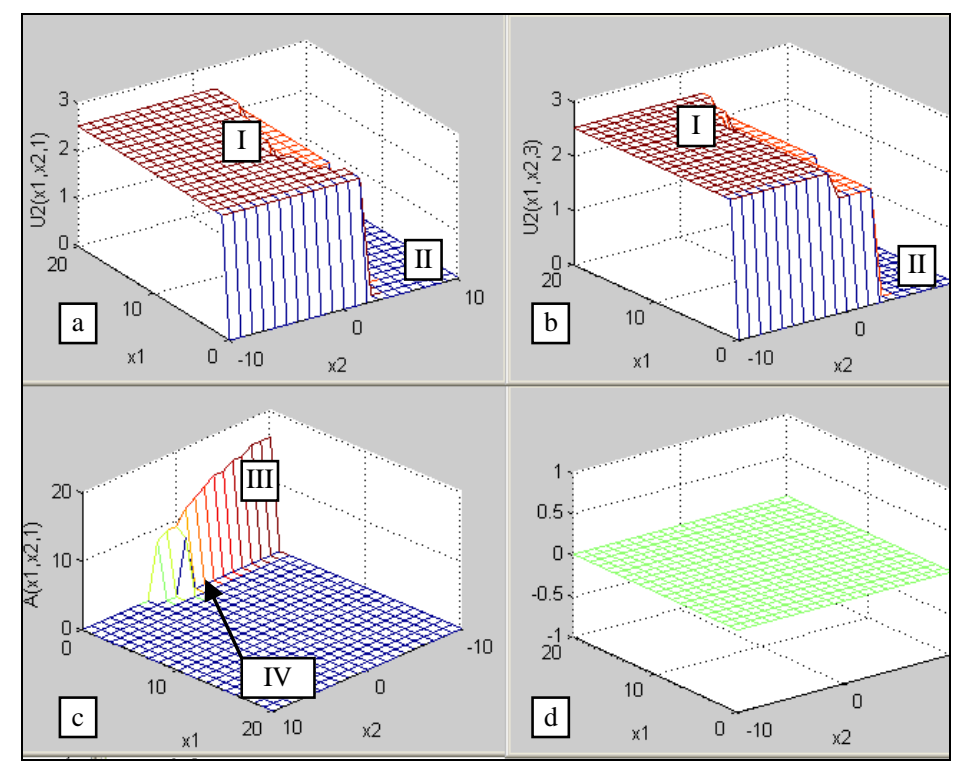

Fig. 2. Optimal production and supply policy

The production and supply policy presented by equations (8) and (9) is completely defined for given values of $\mathrm{s}, \mathrm{Q}$ and $\mathrm{Z}$, called here design factors.

Even if the numerical resolution makes it possible the characterization of the optimal policy, a good approximation remains always a challenge due to implementation difficulties and to the existence of irregularities in the regions boundaries. These irregularities lead sometimes to bad interpretations. In order to ensure the validation of our observations a simulation based approach is proposed in the next section. This approach is aimed at conducting sensitivity analysis leading to a close approximation of the optimal control policy and for determining optimal values of s, $\mathrm{Q}$ and $\mathrm{Z}$.

\section{SIMULATION BASED EXPERIMENTAL APPROACH}

The objective of this section is to find the values of the control policy parameters (design factors) which minimize the incurred cost for the developed control policy. To follow this purpose we will adopt an experimental approach which is a combination of simulation modeling, experimental design and response surface methodology. The reader is referred to Gharbi and Kenne (2003) for more details on the application of this approach in the control of Flexible Manufacturing Systems.

\subsection{Experimental approach}

This approach is presented in Figure 3 and can be summarized by the following main steps:

I. Develop a simulation model to describe the dynamic of the system using the control policy parameterized by the 3 parameters defined previously. These factors are considered as input of such a model and the related incurred cost is defined as its output. Our model was developed using Visual SLAM simulation language (Pritsker and O'Reilly, 1999).

II. Using an experimental design approach, determine the input factors or interactions which have significant effects on the output.

III.Consider the significant factors or interactions as input of a response surface methodology, to fit the relationship between the cost and the input factors. From this estimated relation, the optimal values of the input factors, called $s^{*}, Q^{*}$ and $Z^{*}$ are determined. We refer the reader to Montgomery (2001) for more details on experimental design and response surface methodology approaches. 


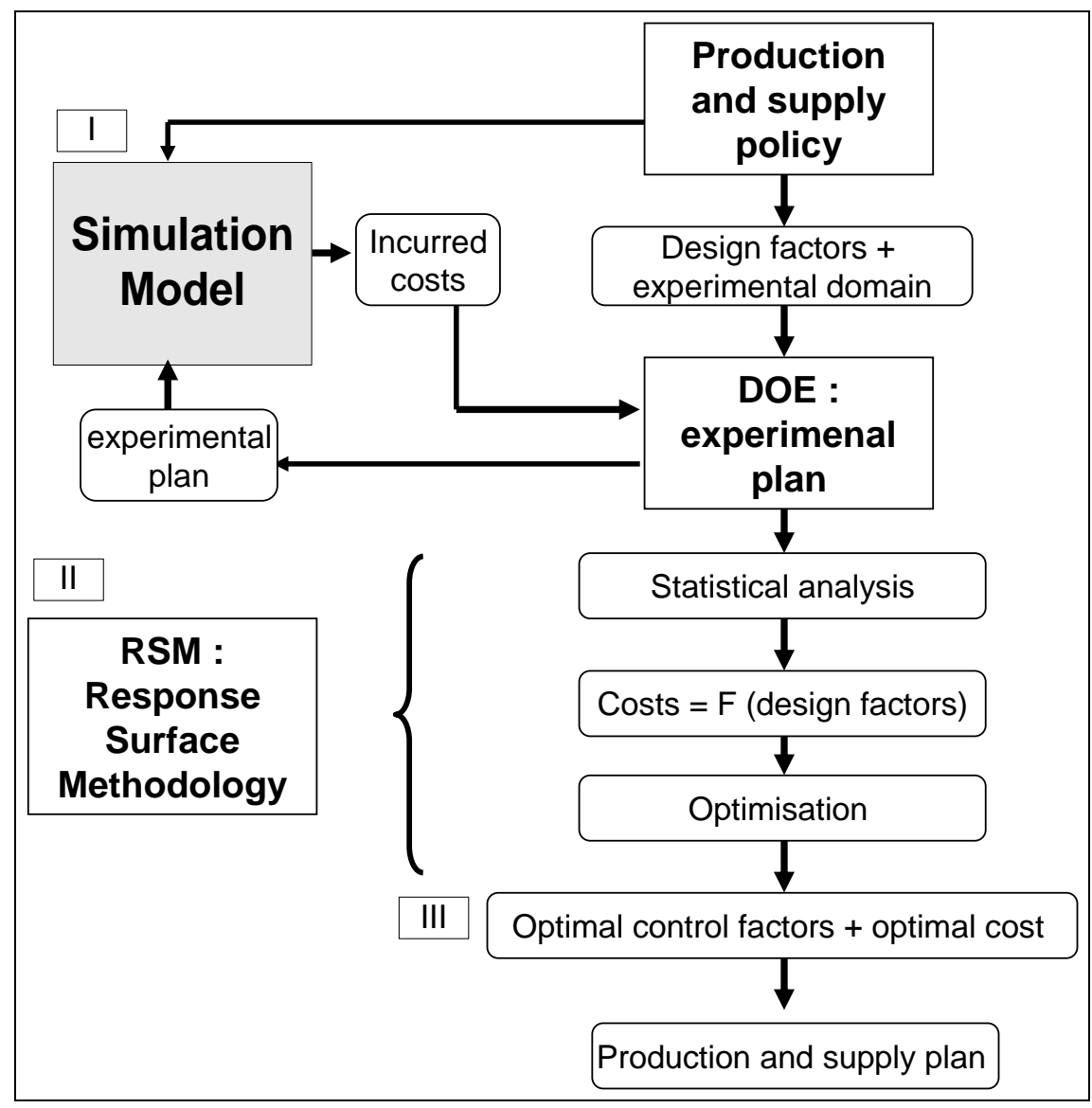

Fig. 3. Experimental approach

\subsection{Simulation model}

The combined simulation model is developed using the Visual SLAM language with C sub-routines. It is interesting to note that the combined discrete/continuous simulation model is more flexible and reduces the execution time (Lavoie et al. (2006)). The Visual SLAM portion is composed of various networks describing specific tasks such as failure and repair events, threshold crossing of inventory variables, etc.... The model is shown at Figure 4 with the following descriptions of the different blocks.

1) The INITIALIZATION block sets the values of, $\mathrm{s}, \mathrm{Q}, Z$, the demand rate, the FMS parameters such as $U_{\max }$, mean time to failure and mean time to repair and the supplier parameters such as the mean time between unavailability and mean time to become available. The maximum and minimum time step specifications for integration of the cumulative variables and allowable errors are also assigned at this step as well as the simulation time $T_{-}$fin and the time for the warm up period.

2) The DEMAND RATE is constant and defined in the INITIALIZATION block. It is shown here as an individual block to facilitate comprehension since it is constantly used as an input in the state equations.

3) The CONTROL POLICY is implemented through the use observation networks that raise a flag whenever one of the thresholds is crossed. The FMS production rate and/or the supply order are then set according to equations (8) and (9).

4) The STATE EQUATIONS are equations (1) defined as a $\mathrm{C}$ language insert. They describe the inventory and backlog variables using the production rates set by the control policy and the binary variables from the failure and repair of the FMS and the availability of the supplier.

5) The AVAILABILITY of the supplier samples the times between unavailability for the supplier. These states of the supplier are incorporated in the state equations by the means of the stochastic process.

6) The FAILURES AND REPAIRS block samples the times to failure and times to repair for the FMS from their respective probability distributions. The operational states of the FMS are incorporated in the state equations by the means of the stochastic process. 


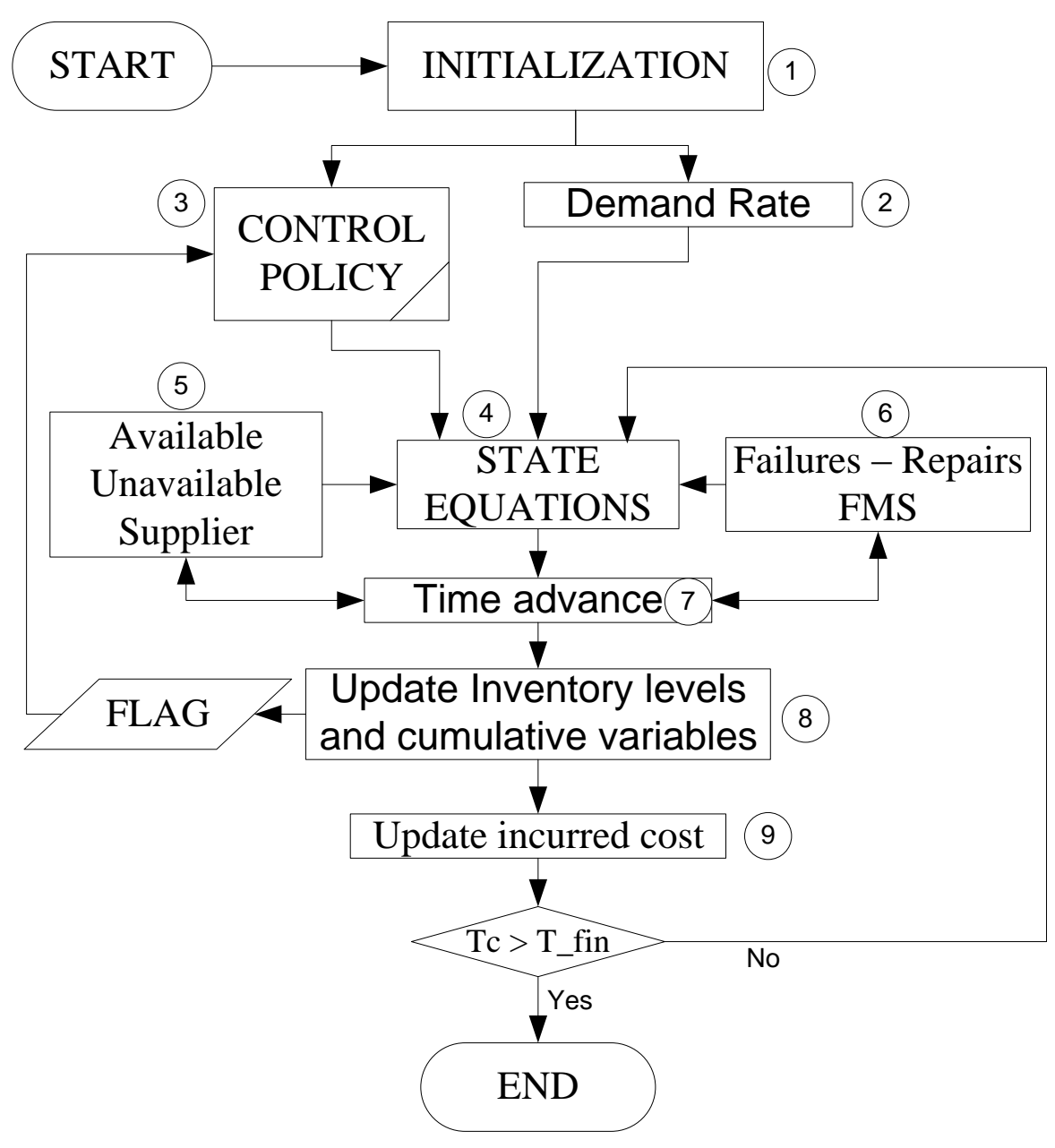

Fig. 4. Simulation block diagram

7) The TIME ADVANCE block uses an algorithm provided by Visual SLAM. It is a combination of discrete event scheduling (failures, repair and unavailability), continuous variable threshold crossing events and time step specifications.

8) The UPDATE INVENTORY LEVELS AND CUMULATIVE VARIABLES block is used once the time step is chosen. The cumulative variables are integrated using the Runge-Kutta-Fehlberg (RKF) method as described in Pritsker \& O'Reilly (1999).

9) The UPDATE INCURRED COST block calculates the incurred cost according to the levels of the different variables and the unit costs.

The simulation ends when current simulation time $T_{c}$ reaches the defined simulation period $T_{-}$fin .

\subsection{Validation of the simulation model}

To verify the accuracy of the model, we verified graphically the dynamics of the stocks to see if the model works according to the control policy given by equations (8) and (9). Figure 5 is a graphical illustration of the trajectories of the raw material
$\left(X_{1}\right)$ and finished products $\left(X_{2}\right)$ stock levels with the following descriptions of the different arrows.

1) Arrow 1 illustrates the maximal production rate that the FMS must follow according to the production policy (8). The FMS produces at this rate to reach the thresh level $\mathrm{Z}$ (equal to 15 in figure 5).

2) Arrow 2 illustrates the production at the demand rate that the FMS must follow according to the production policy (8). The FMS produces at this rate when the thresh level $\mathrm{Z}$ is reached.

3) Arrow 3 and 4 illustrate two failures of the FMS. The first one is repaired before reaching a negative stock level. The second one required more time to be repaired. The FMS became available when $X_{2}=-10$ (see arrow 5). It is interesting to observe that when the FMS is being repaired the raw material level $X_{1}$ keep the same level (see arrow 9).

4) Arrow 6 illustrates a decreasing slope of the raw material level $X_{1}$. When it reaches $s=2$ and according to the order policy given by equation (9) an order quantity $Q=10$ must be send. 


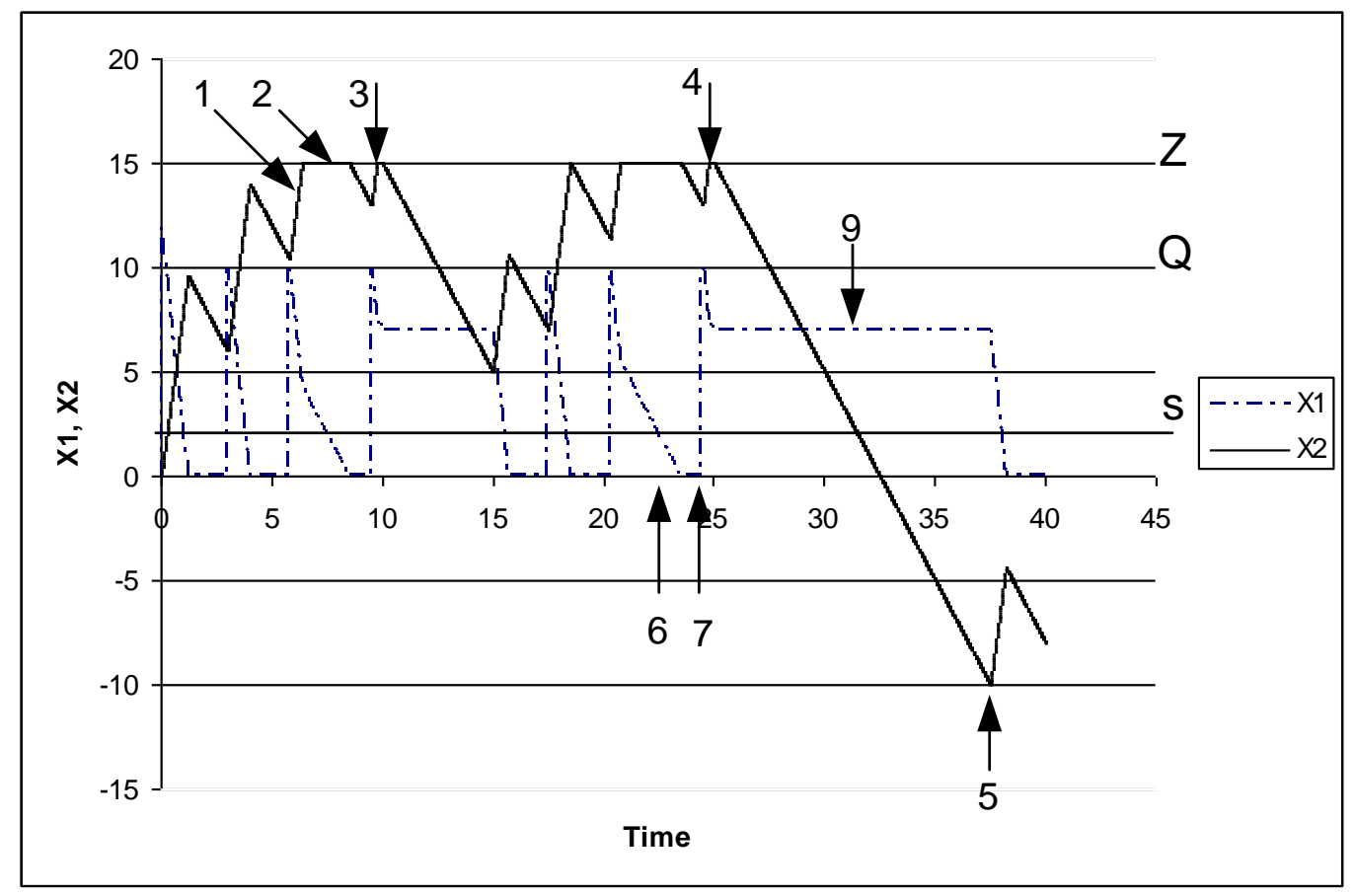

Fig. 5. Stock dynamics

5) Arrow 7 illustrates the reception of the order quantity $Q$. The reception event arrives after a random lead time (time period between arrow 6 and 7).

\subsection{Experimental design}

Three independent variables and one dependent variable (the cost) were considered. The levels of independent variables or design factors should be chosen carefully so that they represent the domain of interest. Due to the convexity property of the value function (6) (Sethi and Zhang, 1994), the first-order response surface model is rejected. In fact, we selected a $3^{3}$ response surface design since we have 3 independent variables at three levels each.

Five replications were conducted for each combination; therefore 135 ( 27 x 5) simulation runs were made. All possible combinations of different levels of factors are provided by the response surface design considered herein. The experimental design is used to study and understand the effects that some parameters, namely s, Q and $\mathrm{Z}$, have on the performance measure (i.e., the cost).

The statistical analysis of the simulation data consists of the multi-factor analysis of the variance (ANOVA). This is done by using statistical software, such as STATGRAPHICS, to provide the effects of the three independent variables on the dependent variable. Table 2 illustrates the ANOVA for the second case of Set IV (see table 3). We can see the significant effect of main factors and interactions (symbol $\mathrm{S}$ in the last column) for the dependent variable, at 0.05 level of significance (i.e., P-value < $0.05)$.

The R-squared value of 0.9627 presented in Table 2, states that $96.27 \%$ of the total variability is explained by the model (Montgomery, 2001). The obtained model includes three main factors (s, Q and Z), and all the interaction and quadratic effects.

Response surface methodology is a collection of mathematical and statistical techniques that are useful for modeling and analysing problems in which a response of interest is influenced by several variables and the objective is to optimize this response (Montgomery, 2001). We assume here that there exists a function $\Phi$ of $\mathrm{s}, \mathrm{Q}$ and $\mathrm{Z}$ that provides the value of the cost corresponding to any given combination of input factors. That is Cost $=\Phi(\mathrm{s}, \mathrm{Q}$, Z).

The function $\Phi($.$) is called the response surface and$ is assumed to be a continuous function of $\mathrm{s}, \mathrm{Q}$ and $\mathrm{Z}$. We choose the second-order model given by:

$$
\begin{aligned}
& \text { Cost }=\beta_{0}+\beta_{11} s+\beta_{12} Q+\beta_{13} Z+\beta_{21} s^{2}+ \\
& \beta_{22} Q^{2}+\beta_{23} Z^{2}+\beta_{31} s \cdot Q+\beta_{32} s . Z+\beta_{33} Z . Q+\varepsilon
\end{aligned}
$$


Where $\mathrm{s}, \mathrm{Q}$ and $\mathrm{Z}$ are the input variables; $\beta_{0}, \beta_{i j}, i, j=1,2,3$ are unknown parameters and $\varepsilon$ is a random error.

From STATGRAPHICS, the estimation of $\beta_{i j}$ is performed and the following nine coefficients achieved. The values of these coefficients for the considered case of the sensitivity analysis are:

$\beta_{0}=2605.4 ; \beta_{11}=-12.02 ; \beta_{12}=-12.54$

$\beta_{13}=-9.36 ; \beta_{21}=0.017 ; \beta_{22}=0.02$

$\beta_{23}=0.013 ; \beta_{31}=0.029 ; \beta_{32}=0.027 ; \beta_{33}=0.02$

The correspondent response surfaces are presented in figure 6,7 . It follows from these figures the optimal values of s, Q and Z, respectively equal to 96,185 and 124

\subsection{Sensitivity analysis}

To illustrate the effect of the costs variations on the design parameters, a sensitivity analysis was conducted. Table 3 details the cost variations and presents the optimal parameters and the incurred optimal cost for the sensitivity analysis cases.

Four sets of cases were conducted, Set I, II, III and IV details respectively the variation of $K, c_{1}^{+}, c_{2}^{+}$and $c_{2}^{-}$. The obtained results, detailed in the next paragraphs, make sense and confirm our expectations.

Table 2 ANOVA table for the total cost, case 2 (Set IV)

Analysis of variance for total Cost 11
\begin{tabular}{|l|l|l|l|l|l|l|}
\hline Source & Sum of Squares & DF & Mean Square & F-Ratio & P-Value & Significant \\
\hline A:s & 169146. & 1 & 169146. & 839.24 & $\mathbf{0 . 0 0 0 0}$ & S \\
\hline B:Q & 102463. & 1 & 102463. & 508.38 & $\mathbf{0 . 0 0 0 0}$ & S \\
\hline C:Z & 95688.0 & 1 & 95688.0 & 474.77 & $\mathbf{0 . 0 0 0 0}$ & S \\
\hline AA & 23400.1 & 1 & 23400.1 & 116.10 & $\mathbf{0 . 0 0 0 0}$ & S \\
\hline AB & 71327.7 & 1 & 71327.7 & 353.90 & $\mathbf{0 . 0 0 0 0}$ & S \\
\hline AC & 113252. & 1 & 113252. & 561.92 & $\mathbf{0 . 0 0 0 0}$ & S \\
\hline BB & 9729.46 & 1 & 9729.46 & 48.27 & $\mathbf{0 . 0 0 0 0}$ & S \\
\hline BC & 32044.3 & 1 & 32044.3 & 158.99 & $\mathbf{0 . 0 0 0 0}$ & S \\
\hline CC & 12851.7 & 1 & 12851.7 & 63.77 & $\mathbf{0 . 0 0 0 0}$ & S \\
\hline blocs & 443.126 & 4 & 110.781 & 0.55 & 0.6996 & NS \\
\hline Total error & 24387.1 & 121 & 201.546 & & & \\
\hline Total (corr.) & 654733. & 134 & & & & \\
\hline
\end{tabular}
R-squared $=\mathbf{9 6 . 2 7 5 3}$ percent(s)

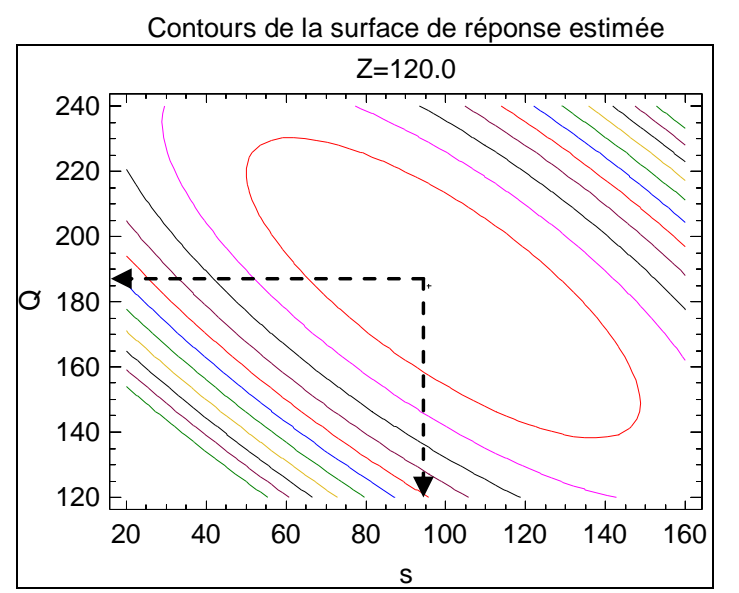

Fig. 6. Cost response surface 1 for case 2 (Set IV).

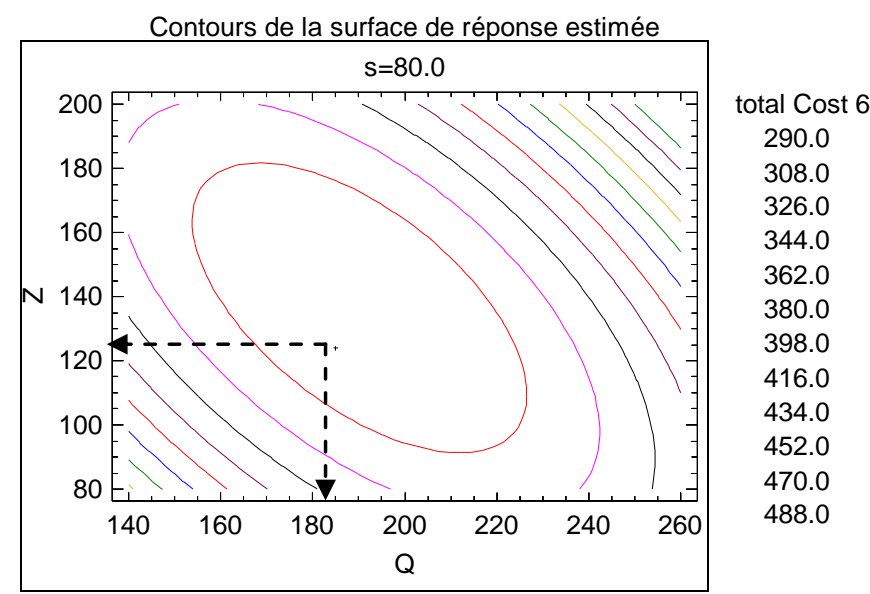

Fig. 7. Cost response surface 2 for case 2 (Set IV). 
- Variation in order cost: when the order cost increases (resp. decreases) the order point decreases (resp. increases), the economic order quantity increases (resp. decreases) and the hedging level (security final stock level) increases (resp. decreases). In deed, when the order cost is higher, one has to order more but less frequently. Moreover, one has to keep a higher level of finished product. This observation shows how the system reacts to transform material to the final stock owing that it incurs the same holding cost than the raw material stock. This reaction insures a higher final security stock level to hedge against future capacity shortages caused by the FMS unavailability.

- Variation in raw material inventory cost: when the inventory cost of raw material increases (resp. decreases), the order point decreases (resp. increases), the order quantity increases (resp. decreases) and the hedging level increases (resp. decreases). This observation shows how the system reacts to transform material to the stock incurring the lowest cost (final stock in this case). This observation is confirmed by the next point.

- Variation in final product inventory cost: when the inventory cost of final product increases (resp. decreases), the order point increases (resp. decreases), the order quantity decreases (resp. increases) and the hedging level decreases (resp. increases). This observation confirms the previous point and show that the dynamic reaction of the FMS makes sense.

- Variation in the final product backlog cost: when the backlog cost increases (resp. decreases) the order point increases (resp. decreases), the economic order quantity decreases (resp. increases) and the hedging level increases (resp. decreases). In deed, when the backlog cost is higher, one has to keep higher stock security levels (i.e., s and Z).

\section{DECISIONAL PROCESS}

In what follows (Figure 8), the quantified feedback policy of the case 2 Set IV of the sensitivity analysis (Table 3, section 4.5) is presented. This illustration shows the actions that should be taken when the FMS is available, and is a function of the stock level of raw material and finished product ( $x_{1}$ and $\left.x_{2}\right)$. The following equations (12 and 13) give the analytical representation of the feedback policy.

$$
\begin{gathered}
U(.):\left\{\begin{array}{ccc}
U_{\max } & \text { if } & X_{2}<124 \\
d & \text { if } & X_{2}=124 \\
0 & \text { if } \quad X_{2}>124
\end{array}\right. \\
A(.):\left\{\begin{array}{c}
185 \text { if } X_{1} \leq 96 \\
0 \quad \text { otherwise }
\end{array}\right.
\end{gathered}
$$

\begin{tabular}{|c|c|c|c|c|c|c|c|c|c|c|}
\hline \multirow{2}{*}{ SETS } & \multirow{2}{*}{ CASES } & \multirow{2}{*}{$K$} & \multirow{2}{*}{$c_{1}^{+}$} & \multirow{2}{*}{$c_{2}^{+}$} & \multirow{2}{*}{$c_{2}^{-}$} & \multicolumn{3}{|c|}{$\begin{array}{c}\text { OPTIMAL } \\
\text { PARAMETERS }\end{array}$} & \multirow{2}{*}{ OTPMAL COST } & \multirow{2}{*}{ IMPACT ON } \\
\hline & & & & & & $s^{*}$ & $Q^{*}$ & $Z *$ & & \\
\hline \multirow{3}{*}{ Set I } & Base case & 100 & 1 & 1 & 40 & 90 & 190 & 117 & 286.73 & ---- \\
\hline & 2 & 150 & 1 & 1 & 40 & 87 & 192 & 118 & 291.95 & $s \downarrow Q \uparrow Z \uparrow$ \\
\hline & 3 & 50 & 1 & 1 & 40 & 93 & 188 & 115 & 281.45 & $s \uparrow Q \downarrow Z \downarrow$ \\
\hline \multirow{3}{*}{ Set II } & Base case & 100 & 1 & 1 & 40 & 90 & 190 & 117 & 286.73 & ---- \\
\hline & 2 & 100 & 1.1 & 1 & 40 & 66 & 195 & 138 & 298.41 & $s \downarrow Q \uparrow Z \uparrow$ \\
\hline & 3 & 100 & 0.9 & 1 & 40 & 117 & 184 & 93 & 273.06 & $s \uparrow Q \downarrow Z \downarrow$ \\
\hline \multirow{3}{*}{ Set III } & Base case & 100 & 1 & 1 & 40 & 90 & 190 & 117 & 286.73 & --- \\
\hline & 2 & 100 & 1 & 1.1 & 40 & 116 & 185 & 89 & 296.30 & $s \uparrow Q \downarrow Z \downarrow$ \\
\hline & 3 & 100 & 1 & 0.9 & 40 & 67 & 194 & 142 & 274.75 & $s \downarrow Q \uparrow Z \uparrow$ \\
\hline \multirow{3}{*}{ Set IV } & Base case & 50 & 1 & 1 & 40 & 93 & 188 & 115 & 281.45 & ---- \\
\hline & 2 & 50 & 1 & 1 & 50 & 96 & 185 & 124 & 290.59 & $s \uparrow Q \downarrow Z \uparrow$ \\
\hline & 3 & 50 & 1 & 1 & 30 & 89 & 192 & 101 & 269.12 & $s \downarrow Q \uparrow Z \downarrow$ \\
\hline
\end{tabular}

$\underline{\text { Table } 3 \text { Sensitivity analysis data and results }}$ 


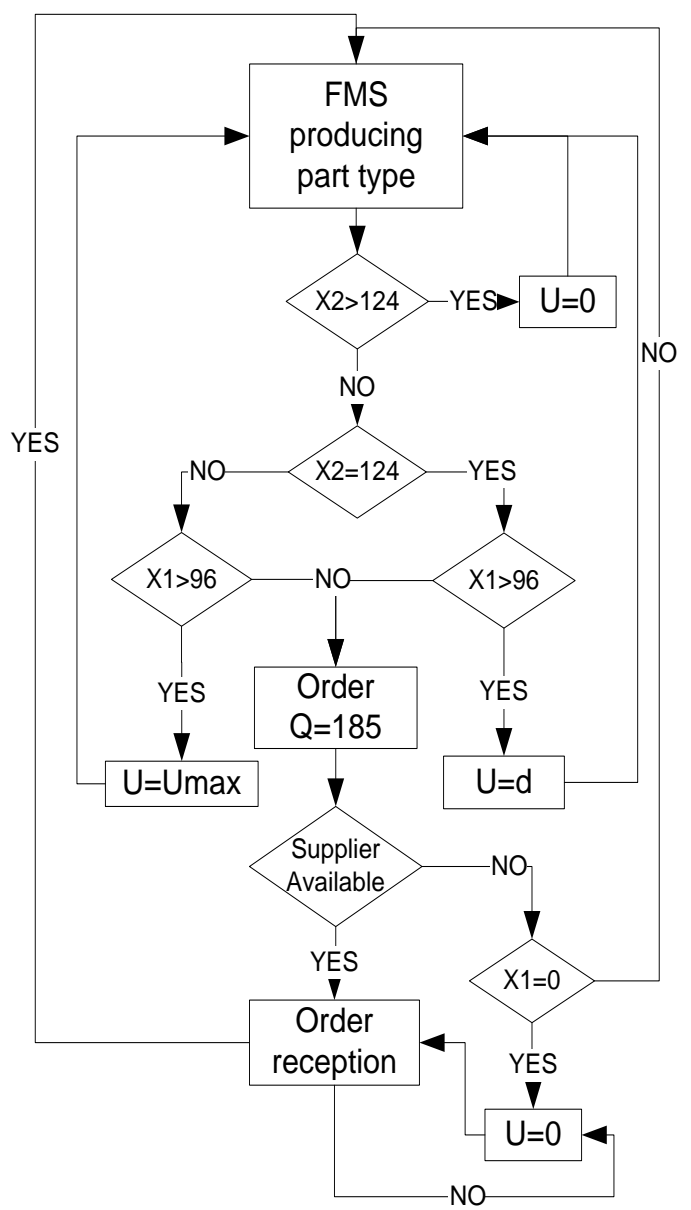

Fig. 8. logigram of the considered case

\section{CONCLUSION}

In this paper, we have developed a complete and parameterized production and supply policy for the three stages flexible manufacturing system. We have solved numerically the Hamilton Jacobi Bellman equations of the problem and carried out deep experimentation and sensitivity analysis. The obtained control policy has been shown to be described by a combined HPP and (s, Q) policies defined by three parameters. To optimize the proposed heuristic parameters and so, to achieve a close approximation of the optimal production and supply policy, an experimental approach based on design of experiment, simulation modeling and response surface methodology has been presented.

For large manufacturing systems control problems, we think that adopting the modelling approach presented in this paper may conduct to satisfactory results. To deal with the complexity behind the application of the same approach for larger systems, fractional experimental design could be good alternatives.

\section{REFERENCES}

Akella, R. and P. R. Kumar (1986). Optimal control of production rate in a failure-prone manufacturing system. IEEE. Trans. Automat. Contr., AC-31, 2, pp. 116-126.

Bensoussan, A., M. Crouhy and J. M. Proth (1983). Mathematical theory of production planning, New York : North-Holland, New York.

Boukas, E. K. and A. Haurie (1990). Manufacturing flow control and preventive maintenance: a stochastic control approach. IEEE. Trans. Automat. Contr., 35, 9, pp. 1024-1031.

Cheng, F. and S. P. Sethi (1999). Optimality of state dependent $(\mathrm{s}, \mathrm{S})$ policies in inventory models with markov modulated demand and lost sales. Production and operations management, 8, 2, pp. 183-192.

Feng, Y. and H. Yan (2000). Optimal production control in a discrete manufacturing system with unreliable machines and random demands. IEEE. Trans. Automat. Cont., 45, 12, pp. 2280-2296.

Gharbi, A. and J. P. Kenne (2003). Optimal production control problem in stochastic multiple product multiple machine manufacturing system. IIE transactions, 35, pp. 941-952.

Gullu, R., E. Onol and N. Erkip (1999). Analysis of an inventory system under supply uncertainty, Int. J. Production Economics, 59, pp. 377-385.

Hajji, A., A. Gharbi and J. P. Kenne (2004). Production and setup control of a failure prone manufacturing system. International Journal of Production Research, 42, 6, pp. 1107-1130.

Kimemia, J. G. and S. B. Gershwin (1983). An algorithm for the computer control production in flexible manufacturing systems. IIE transactions, 15, pp. 353-362.

Kushner, H. J. and P. G. Dupuis (1992). Numerical methods for stochastic control problems in continuous time. Springer-verlag, NY.

Lavoie, P., J. P. Kenne and A. Gharbi (2006). Production control and combined discrete/continuous simulation modeling in failure prone transfer lines. International Journal of Production Research, to appear.

Lee, W. (2005). A joint economic lost size model for raw material ordering, manufacturing setup, and finishing goods delivering. Omega, 33, pp. 163174.

Montgomery, D. C. (2001). Design and analysis of experiments. John Wiley \& Sons, NY.

Pritsker, A. A. B. and J. J. O'Reilly (1999). Simulation with Visual SLAM and Awesim. John Wiley \& Sons, NY.

Sethi, S. P. and G. L. Thompson (2000). Optimal control theory: application to management science and economics. Kluwer Academic Publishers, Boston.

Sethi, S. P. and Q. Zhang (1994). Hierarchical decision making in stochastic manufacturing systems. Birkhauser, Boston. 